\section{Association of sonographic features and clinicopathologic factors of papillary thyroid microcarcinoma for prevalence of lymph node metastasis: a retrospective analysis}

Quan Zou'

https://orcid.org/0000-0003-2944-8717

Sumei $\mathrm{Ma}^{1}$

https://orcid. org/0000-0002-9133-801X

Xinghu Zhou ${ }^{2}$

hitps://orcid.org/0000-0001-9920-9231

\begin{abstract}
Objective: The objective of the study was to develop an association between clinicopathologic and sonographic features of patients with papillary thyroid microcarcinoma and the prevalence of lymph node metastasis. Subjects and methods: Clinicopathologic and sonographic features of 415 patients of papillary thyroid microcarcinoma with $(n=102)$ or without $(n=313)$ lymph node metastasis were retrospectively reviewed. The thickness of the lymph node $\geq 6 \mathrm{~mm}$ with intra-lymph nodal occupying lesions considered lymph node metastasis. Also, it was considered metastasis if lymph node perfusion or blood flow defect was found with any thickness size. Univariate following multivariate analysis was performed for the prediction of sonographic features and clinicopathologic factors for the prevalence of lymph node metastasis. Results: Male gender $(p=0.041)$, age $<45$ years $(p=0.042)$, preoperative calcitonin $>65 \mathrm{pg} / \mathrm{mL}(p=0.039)$, nodule size $>5 \mathrm{~mm}$ in diameter $(p=$ $0.038)$, bilaterality $(p=0.038)$, tumor capsular invasion $(p=0.048)$, cystic change $(p=0.047)$, and hyper vascularity $(p=0.049)$ of thyroid nodules were associated with lymph node metastasis. Also, thyroid nodules $5 \mathrm{~mm}$ and more in diameter may have high aggressiveness. Conclusion: These data helped the surgeon for individualized treatment in thyroid carcinoma and avoid unnecessary prophylactic surgery of the lymph node. Arch Endocrinol Metab. 2020;64(6):803-9
\end{abstract}

\section{Keywords}

Clinicopathologic factors; lymph node metastasis; lymph node resection; papillary thyroid microcarcinoma; sonographic features; thyroidectomy
1 Department of Ultrasound, The First Hospital of Lanzhou University, Lanzhou, China ${ }^{2}$ Department of Cardiology, The First Hospital of Lanzhou University, Lanzhou, China
Correspondence to:

Quan Zou

Department of Ultrasound,

The First Hospital of Lanzhou

University,

Lanzhou 730000, China

brigittefitzpatrickpqi@yahoo.com

Received on May/20/2020

Accepted on Aug/6/2020

DOI: 10.20945/2359-3997000000297

\section{INTRODUCTION}

$\mathrm{T}$ hyroid carcinoma measuring $1 \mathrm{~cm}$ or less in its greatest dimension is considered as thyroid microcarcinoma (1). Papillary thyroid microcarcinoma is the most common form of thyroid microcarcinoma (2). It is associated with the risk of lymph node metastasis $(1,3)$ and exhibits aggressive behavior (2). However, low-risk papillary microcarcinomas have excellent oncological outcomes of active surveillance (4). Papillary thyroid microcarcinoma is a subset of tumors with an indolent course and an even less aggressive treatment now proposed for these patients. However, some of these tumors may have higher rates of recurrent and persistent disease. Therefore, it is important to identify initial clinical and pathological characteristics that can predict a higher risk of progressive disease, avoiding undertreatment in this scenario. This is especially important for surgeons and clinical endocrinologists who face an increase in the incidence of papillary thyroid microcarcinoma today and must make accurate and economical treatment decisions in their routine.

Ultrasound imaging and fine-needle aspiration cytopathology are generally used for the diagnosis of papillary thyroid microcarcinoma (5). Ultrasound evaluates half of the lymph nodes due to the presence of thyroid (1). A retrospective chart review reported only 38\% sensitivity of high-resolution ultrasound for predicting lymph node metastasis in papillary thyroid carcinoma (6). Japanese Society of Thyroid Surgeons 
(JSTS) (7) and Chinese Society of Clinical Oncology (CSCO) (8) guidelines suggested prophylactic lymph node resection to overcome complications regarding reoperations $(8,9)$ but it is controversial in patients with papillary thyroid microcarcinoma (10) because of no evidence that rates of recurrence are decreased with this prophylactic lymph node resection (11). A retrospectively studies reported that lymph node metastasis frequency is higher in multifocal papillary thyroid microcarcinoma with higher sized nodules $(1,5,10)$. The other retrospective study reported that clinicopathologic factors of papillary thyroid microcarcinoma of 5 or less $\mathrm{mm}$ diameter $(\varnothing)$ nodules were less aggressive than more than $5 \mathrm{~mm} \emptyset$ nodules (2). A retrospectively studies reported that preoperative more than $65 \mathrm{pg} / \mathrm{mL}$ serum levels of calcitonin, subcapsular locations and the size of nodules are associated with lymph node metastasis $(6,10)$. While retrospective studies reported that preoperative locations and the size of nodules are not associated with lymph node metastasis $(2,5)$. Moreover, papillary thyroid microcarcinoma patients with clinically negative lymph node cancer have reported $3 \%$ lymph node metastasis in the follow-up period after surgeries (12). Therefore, it is advisable to predict the association of clinicopathologic factors and preoperative sonographic features for papillary thyroid microcarcinoma for improvement of the diagnostic value of ultrasonography (2).

The aim of the retrospective study was to develop an association between clinicopathologic and sonographic features of patients with papillary thyroid microcarcinoma and the prevalence of lymph node metastasis.

\section{SUBJECTS AND METHODS}

\section{Ethics approval and consent to participate}

The first hospital of Lanzhou University, China approved the retrospective study (No. L-296) and waived the requirements of written informed consent form from the enrolled patients. Electronic medical records of patients have studied anonymously.

\section{Study population}

From 15 January 2016 to 28 November 2019, a total of 2,830 patients underwent thyroid surgery (total thyroidectomy, lobectomy, or central compartment lymph node resection) at the first hospital of Lanzhou University, Lanzhou, China. Patients age $\geq 18$ years, surgical pathological diagnosis reported papillary thyroid microcarcinoma $(10 \mathrm{~mm}$ or less than in its maximum $\varnothing)(9)$ with or without lymph node metastasis were retrospectively reviewed. 2,407 patients had other diagnosis than papillary thyroid microcarcinoma, two patients had incomplete data, one patient had age less than 18 years, two patients had a history of radiation exposure, three patients had a family history of thyroid carcinoma. Therefore, these patients were excluded from the study. In the case of multifocality, the largest nodule was used for analysis (5). Sonographic and clinicopathological data of 415 patients were used for analysis (Figure 1).

\section{Ultrasonography}

Grayscale and power Doppler ultrasound performed using ultrasound equipment (iU22, Philips Medical Systems, Amsterdam, Netherlands) with $7 \mathrm{MHz}$ linear transducers. Cervical and thyroid sonography was performed in the longitudinal, transverse, and oblique planes. Ultrasound was performed by radiologists (minimum 3-years' experience) of the institute. All ultrasound images were analyzed by ultrasound technologists (had 5-years of experience in thyroid imaging).

\section{Histopathology}

It was performed for fresh surgically resected nodules of all patients by the pathologists (minimum 3-years' experience) of the institute as per the 2004 World Health Organization (WHO) criteria. If two foci were found on at least one lobe it was considered bilaterality. $\varnothing$ was calculated as the average of maximal diameter of all sides of nodules (1). The accompanying disease was considered Hashimoto's thyroiditis (2). Clinicopathologic parameters were collected by authors.

\section{Lymph node metastasis}

After surgeries in follow-up, in grayscale or color Doppler ultrasound, the thickness of the lymph node $\geq 6 \mathrm{~mm}$ with intra-lymph nodal occupying lesions considered lymph node metastasis. Also, it was considered metastasis if lymph node perfusion or blood flow defect was found with any thickness size. If perfusion or blood flow defect found with any size thickness of the lymph node considered as metastasis (13). The suspected lymph nodes been submitted to fine needle aspiration for cytopathological confirmation. The decision of lymph node metastasis was reached by ultrasound technologists. 


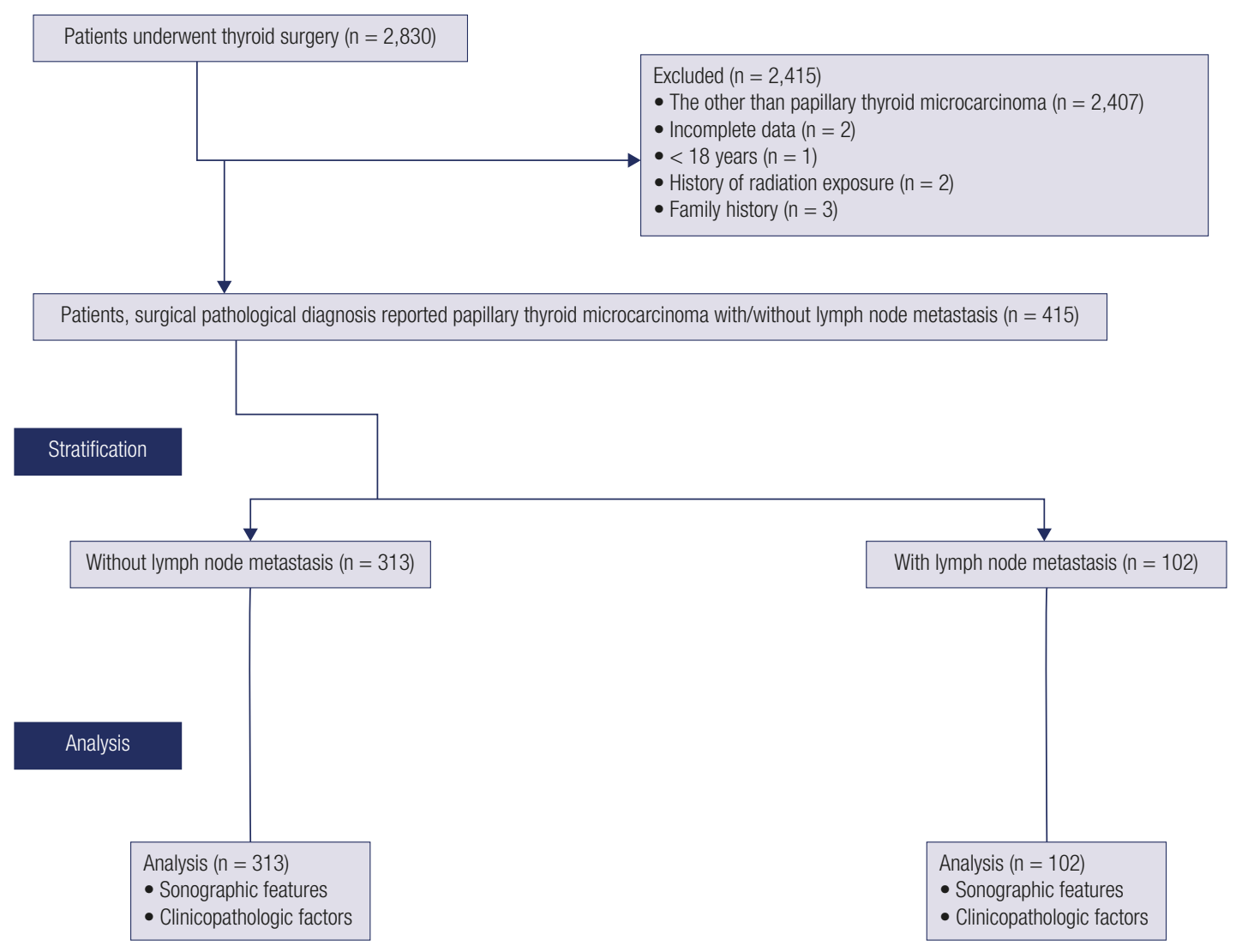

Figure 1. Retrospective study analysis chart.

\section{Statistical analysis}

SPSS v25.0 IBM Incorporation, Armonk, NY, United States was used for statistical analysis purposes. Constant data demonstrate frequency (percentage) and continuous data demonstrate mean \pm SD. Fischer exact test for constant data and twotailed unpaired $t$-test for continuous data performed for statistical analysis (1). Univariate following multivariate analysis was performed for the prediction of sonographic features and clinicopathologic factors for the prevalence of lymph node metastasis (3). All the results were considered significant at a $95 \%$ confidence level.

\section{RESULTS}

\section{Sonographic features}

A minimum of 1 nodule/patient and a maximum of 3 nodules/patients were reported. The other ultrasound parameters are reported in Table 1. A total of 102 patients were developed lymph node metastasis in the follow-up time (the thickness of the lymph node $\geq 6 \mathrm{~mm}$ with intra-lymph nodal occupying lesions: 57 patients, lymph node perfusion: 37 patients, and blood flow defect: 8 patients).

\section{Clinicopathologic factors}

Among 415 patients 299 patients were female and 116 patients were male. The other demographical and clinicopathologic factors of patients are reported in Table 2.

\section{Association of features for the prevalence of lymph node metastasis}

Univariate analysis reported that tumor capsular invasion $(p<0.0001)$, cystic change $(p=0.001)$, and hypervascularity $(p=0.015)$ of thyroid nodules were associated with lymph node metastasis (Table 3 ). 
Table 1. The preoperative ultrasound features of the enrolled patients

\begin{tabular}{|c|c|c|}
\hline \multicolumn{2}{|l|}{ Characteristics } & $\begin{array}{l}\text { Population/ } \\
\text { value }\end{array}$ \\
\hline \multicolumn{2}{|c|}{ Data of patients included in the analysis } & 415 \\
\hline \multirow[t]{3}{*}{ Nodules/patient } & Minimum & 1 \\
\hline & Maximum & 3 \\
\hline & Mean \pm SD & $1.21 \pm 0.25$ \\
\hline \multirow[t]{3}{*}{ Distance to the carotid artery ( $\mathrm{mm})$} & Minimum & 5.81 \\
\hline & Maximum & 27.61 \\
\hline & Mean \pm SD & $11.92 \pm 3.15$ \\
\hline \multirow[t]{3}{*}{ Depth } & Minimum & 4.11 \\
\hline & Maximum & 23.12 \\
\hline & Mean \pm SD & $10.15 \pm 4.15$ \\
\hline \multirow[t]{2}{*}{ Tumor capsular invasion } & Yes & $79(19)$ \\
\hline & No & $336(81)$ \\
\hline \multirow[t]{4}{*}{ Location of tumor } & Right lobe & $165(40)$ \\
\hline & Left lobe & $90(22)$ \\
\hline & Isthmus & $17(4)$ \\
\hline & Multicentric & $143(34)$ \\
\hline \multirow[t]{2}{*}{ Ratio of length/width } & $<1$ & $141(34)$ \\
\hline & $\geq 1$ & $274(66)$ \\
\hline \multirow[t]{2}{*}{ Boundary } & Clear & $59(14)$ \\
\hline & Unclear & $356(86)$ \\
\hline \multicolumn{2}{|l|}{ Peripheral halo ring } & $22(5)$ \\
\hline \multicolumn{2}{|l|}{ Hypoechogenicity } & $94(23)$ \\
\hline \multicolumn{2}{|l|}{ Isoechogenicity } & $21(5)$ \\
\hline \multicolumn{2}{|l|}{ Hyperechogenicity } & $5(1)$ \\
\hline \multicolumn{2}{|l|}{ Cystic change } & $29(7)$ \\
\hline \multicolumn{2}{|l|}{ Microcalcification } & $248(60)$ \\
\hline \multicolumn{2}{|l|}{ Macrocalcification } & $28(7)$ \\
\hline \multicolumn{2}{|l|}{ Normal vascularity } & $227(55)$ \\
\hline \multicolumn{2}{|l|}{ Hyper vascularity } & $61(15)$ \\
\hline
\end{tabular}

Constant data demonstrate frequency (number) and continuous data demonstrate mean \pm SD.

Univariate analysis reported that male gender $(p<$ $0.0001)$, age $<45$ years $(p=0.011)$, and preoperative calcitonin level $>65 \mathrm{pg} / \mathrm{mL}(p<0.0001)$, nodule $\varnothing$ $>5 \mathrm{~mm}(p<0.0001)$, and multifocality of nodules $(p<0.0001)$ were associated with lymph node metastasis (Table 4).

Multivariate analysis reported that male gender $(p=0.041)$, age $<45$ years $(p=0.042)$, preoperative calcitonin $>65 \mathrm{pg} / \mathrm{mL}(p=0.039)$, nodule size $>$ $5 \mathrm{~mm} \varnothing(p=0.038)$, bilaterality $(p=0.038)$, tumor capsular invasion $(p=0.048)$, cystic change $(p=$ $0.047)$, and hyper vascularity $(p=0.049)$ of thyroid nodules were associated with lymph node metastasis (Table 5).
Table 2. The demographical and clinicopathologic factors of the enrolled patients

\begin{tabular}{llc}
\hline Characteristics & Female & $\begin{array}{c}\text { Population/ } \\
\text { value }\end{array}$ \\
\hline Data of patients included in the analysis & $\mathbf{4 1 5}$ \\
\hline Gender & Male & $299(72)$ \\
& $<45$ & $116(28)$ \\
Age (years) & $\geq 45$ & $181(44)$ \\
& Mean \pm SD & $234(56)$ \\
Lymph node metastasis & Central lymph node metastasis & $49.12 \pm 8.47$ \\
in follow up after surgery & Lateral lymph node metastasis & $21(20)$ \\
Body mass index (kg/m²) & & $24.81 \pm 1.85$ \\
Ethnicity & Han Chinese & $376(91)$ \\
& Mongolian & $34(8)$ \\
Preoperative calcitonin & $\leq 65$ pg/ mL & $5(1)$ \\
& $>65$ pg/ mL & $187(45)$ \\
Nodule size (mm; & $\leq 5$ & $228(55)$ \\
diameter) & $>5$ & $176(42)$ \\
& Mean \pm SD & $239(58)$ \\
Multifocality & Unilaterality & $6.01 \pm 0.35$ \\
\hline Hashimoto's thyroiditis & Bilaterality & $261(63)$ \\
& & $154(37)$ \\
& & $94(23)$ \\
& & $15.12 \pm 8.12$ \\
\hline
\end{tabular}

Constant data demonstrate frequency (number) and continuous data demonstrate mean $\pm \mathrm{SD}$.

\section{DISCUSSION}

The study reported that tumor capsular invasion, cystic change, and hypervascularity of thyroid nodules were independent ultrasound features for the predictor of lymph node metastasis. The results of the study were agreed with the results of retrospective studies $(2,3,6,10)$. Extra thyroidal extension of nodules (5) and multifocal lesions (1) have chances of lymph node metastasis. Calcification and vascularity increased as size increased (2). Preoperative ultrasound plays an important role in the prediction and management of lymph node metastasis in patients with papillary thyroid microcarcinoma.

The study reported that the male gender, age $<45$ years, preoperative calcitonin $>65 \mathrm{pg} / \mathrm{mL}$, nodule size $>5 \mathrm{~mm} \varnothing$, and bilaterality of nodules were independent clinicopathological parameters for the predictor of lymph node metastasis. The results of the study were agreed with the results of retrospective studies $(1-3,5,9,10)$. Thyroid nodules $>5 \mathrm{~mm} \varnothing$ have 
Table 3. Univariate analysis for association of sonographic features for the prevalence of lymph node metastasis

\begin{tabular}{|c|c|c|c|c|}
\hline \multicolumn{2}{|l|}{ Characteristics } & No lymph node metastasis & $\begin{array}{l}\text { Lymph node metastasis in } \\
\text { follow up after surgery }\end{array}$ & Comparisons \\
\hline \multicolumn{2}{|c|}{ Data of patients included in the analysis } & 313 & 102 & $p$-value \\
\hline \multirow[t]{3}{*}{ Distance to the carotid artery $(\mathrm{mm})$} & Minimum & 5.81 & 6.01 & 0.054 \\
\hline & Maximum & 27.61 & 25.12 & \\
\hline & Mean \pm SD & $11.12 \pm 4.15$ & $12.01 \pm 3.69$ & \\
\hline \multirow[t]{3}{*}{ Depth } & Minimum & 4.11 & 5.01 & 0.298 \\
\hline & Maximum & 22.99 & 23.12 & \\
\hline & Mean \pm SD & $10.03 \pm 3.39$ & $10.45 \pm 4.01$ & \\
\hline \multirow[t]{2}{*}{ Tumor capsular invasion } & 'Yes & $34(11)$ & $45(44)$ & $<0.0001$ \\
\hline & No & $279(89)$ & $57(56)$ & \\
\hline \multirow[t]{4}{*}{ Location of tumor } & Right lobe & $134(43)$ & $31(30)$ & 0.104 \\
\hline & Left lobe & $68(22)$ & $22(22)$ & \\
\hline & Isthmus & $12(4)$ & $5(5)$ & \\
\hline & Multicentric & $99(32)$ & $44(43)$ & \\
\hline \multirow[t]{2}{*}{ Ratio of length/width } & $<1$ & $108(35)$ & 33 (32) & 0.719 \\
\hline & $\geq 1$ & $205(65)$ & $69(68)$ & \\
\hline \multirow[t]{2}{*}{ Boundary } & Clear & $44(14)$ & $15(15)$ & 0.871 \\
\hline & Unclear & $269(86)$ & $87(85)$ & \\
\hline \multicolumn{2}{|l|}{ Peripheral halo ring } & $16(5)$ & $6(6)$ & 0.798 \\
\hline \multicolumn{2}{|l|}{ Hypoechogenicity } & $72(23)$ & $22(22)$ & 0.892 \\
\hline \multicolumn{2}{|l|}{ Isoechogenicity } & $16(5)$ & $5(5)$ & 0.584 \\
\hline \multicolumn{2}{|l|}{ Hyperechogenicity } & $4(1)$ & $1(1)$ & 0.998 \\
\hline \multicolumn{2}{|l|}{ Cystic change } & $14(5)$ & $15(15)$ & 0.001 \\
\hline \multicolumn{2}{|l|}{ Microcalcification } & $183(59)$ & $65(64)$ & 0.356 \\
\hline \multicolumn{2}{|l|}{ Macrocalcification } & $19(6)$ & $9(9)$ & 0.364 \\
\hline \multicolumn{2}{|l|}{ Normal vascularity } & $173(55)$ & $54(53)$ & 0.732 \\
\hline \multicolumn{2}{|l|}{ "Hyper vascularity } & $38(12)$ & $23(23)$ & 0.015 \\
\hline
\end{tabular}

Constant data demonstrate frequency (number) and continuous data demonstrate mean \pm SD. Fischer exact test for constant data and two-tailed unpaired $t$-test for continuous data performed for statistical analysis. A $p<0.05$ considered significant. . Significant parameter for lymph node metastasis.

Table 4. Univariate analysis for association of clinicopathologic factors for prevalence of lymph node metastasis

\begin{tabular}{|c|c|c|c|c|}
\hline \multicolumn{2}{|l|}{ Characteristics } & No lymph node metastasis & $\begin{array}{l}\text { Lymph node metastasis in } \\
\text { follow up after surgery }\end{array}$ & Comparisons \\
\hline \multicolumn{2}{|c|}{ Data of patients included in the analysis } & 313 & 102 & $p$-value \\
\hline \multirow[t]{2}{*}{ Gender } & Male ${ }^{*}$ & $46(15)$ & $70(69)$ & $<0.0001$ \\
\hline & Female & 267 (85) & $32(31)$ & \\
\hline \multirow[t]{3}{*}{ Age (years) } & $<45^{*}$ & $125(40)$ & $56(55)$ & 0.011 \\
\hline & $\geq 45$ & $188(60)$ & $46(45)$ & \\
\hline & Mean \pm SD & $54.12 \pm 10.12$ & $47.52 \pm 7.12$ & \\
\hline Body mass index (kg/m²) & & $25.02 \pm 1.95$ & $24.59 \pm 2.11$ & 0.059 \\
\hline \multirow[t]{3}{*}{ Ethnicity } & Han Chinese & $286(91)$ & $90(88)$ & 0.562 \\
\hline & Mongolian & $24(8)$ & $10(10)$ & \\
\hline & Tibetan & $3(1)$ & $2(2)$ & \\
\hline \multirow[t]{2}{*}{ Preoperative calcitonin } & $\leq 65 \mathrm{pg} / \mathrm{mL}$ & $180(58)$ & $7(7)$ & $<0.0001$ \\
\hline & *> $65 \mathrm{pg} / \mathrm{mL}$ & $133(42)$ & $95(93)$ & \\
\hline \multirow[t]{3}{*}{ Nodule size (mm; diameter) } & $\leq 5$ & 109 (35) & $67(66)$ & $<0.0001$ \\
\hline & $>5^{\star}$ & 204 (65) & $35(34)$ & \\
\hline & Mean \pm SD & $5.45 \pm 0.25$ & $7.15 \pm 0.55$ & \\
\hline \multirow[t]{2}{*}{ Multifocality } & Unilaterality & 227 & 34 & $<0.0001$ \\
\hline & Bilaterality* & 86 & 68 & \\
\hline Hashimoto's thyroiditis & & $69(22)$ & $25(25)$ & 0.589 \\
\hline
\end{tabular}

Constant data demonstrate frequency (number) and continuous data demonstrate mean \pm SD. Fischer exact test for constant data and two-tailed unpaired $t$-test for continuous data performed for statistical analysis. A $p<0.05$ considered significant. * Significant parameter for lymph node metastasis. 
Table 5. Association of parameters with lymph node metastasis

\begin{tabular}{|c|c|c|c|}
\hline Data of patients included in the analysis & & 102 & \\
\hline Parameters & Odd ratio & $95 \%$ confidence interval & $p$-value \\
\hline Gender ( ${ }^{*}$ male vs. female) & 0.781 & $0.625-0.965$ & 0.041 \\
\hline $\operatorname{Age}\left(<45^{\star}\right.$ years $v s . \geq 45$ years $)$ & 0.778 & $0.642-0.972$ & 0.042 \\
\hline Preoperative calcitonin (> 65* pg/ mL vs. $\leq 65 \mathrm{pg} / \mathrm{mL}$ ) & 0.762 & $0.632-0.985$ & 0.039 \\
\hline Nodule size $\left(>5^{\star} \mathrm{mm} \emptyset\right.$ vs. $\left.\leq 5 \mathrm{~mm} \emptyset\right)$ & 0.752 & $0.629-0.987$ & 0.038 \\
\hline Multifocality (*Bilaterality vs. Unilaterality) & 0.743 & $0.665-0.998$ & 0.038 \\
\hline Tumor capsular invasion ("yes vs. no) & 0.779 & $0.685-0.985$ & 0.048 \\
\hline Cystic change (*yes vs. no) & 0.778 & $0.665-0.954$ & 0.047 \\
\hline Vascularity (*hyper vascularity vs. no vascularity) & 0.776 & $0.663-0.952$ & 0.049 \\
\hline
\end{tabular}

Multivariate analysis. Data of absence lymph node metastasis considered the reference standard. A $p<0.05$ considered significant. * ${ }^{*}$ ignificant parameter for lymph node metastasis. $\emptyset$ : Diameter.

high aggressiveness (2). Like radiological features, the clinicopathological characters also helpful for further thyroidectomy or radioiodine ablation.

The study used a $5 \mathrm{~mm} \varnothing$ threshold for the prediction of lymph node metastasis. The American Thyroid Association (ATA) guidelines recommended 5 $\mathrm{mm} \varnothing$ nodules for fine-needle aspiration biopsies (14). Papillary thyroid microcarcinoma changes little in size during a long follow-up (15). Therefore, the study used a $5 \mathrm{~mm} \varnothing$ nodule threshold to predict the aggressiveness of nodules for the prediction of lymph node metastasis.

The study recommended careful prophylactic surgery of lymph node in specific clinicopathological and sonographic features but has several limitations, for example, lack of a randomized case-control trial, separate parameters are required to evaluate for central and lateral lymph node metastasis. Follow-up data are not presented. The issue of generalizability because lymph node dissection is performed in China but not routinely performed in Western countries (1). A retrospective study reported that Tumor-node-metastasis (TNM) III and IV are associated with lymph node metastasis (9) but the current study did not evaluate the association of TNM staging for the association of lymph node metastasis. The possible justification is that it is not a surprising finding. As large numbers of surgeries and surgeons were involved. The degree of expertise also has effects on management of thyroidectomy. How many patients underwent lymph node dissection and what was rate of lymph node metastasis among these was not reported. Association between postoperative recurrence and lymph node metastasis did not report. The histopathological diagnosis of the tumor, classic, follicular, tall cell variant, and their an association with medullary carcinoma did not perform. All ultrasound images were not performed by ultrasound technologists but all ultrasound images were analyzed by ultrasound technologists. As being a retrospective study is known that histopathology was not performed by the same pathologist and it was not reviewed by a second or third pathologist.

In conclusion, besides the available guidelines, the retrospective study reported that the male gender, age $<45$ years, preoperative calcitonin $>65 \mathrm{pg} / \mathrm{mL}$, bilaterality, capsular invasion, cystic change, and hypervascularity of thyroid nodules associated with lymph node metastasis. Also, thyroid nodules $5 \mathrm{~mm}$ and more in diameter may have high aggressiveness. These data helped the surgeon for individualized treatment in thyroid carcinoma and avoid unnecessary prophylactic surgery of the lymph node. Chinese Society of Clinical Oncology diagnosis and treatment guidelines for thyroid cancer management is required to update.

Acknowledgments: the authors are thankful for the radiological, medical, and pathological staff of the first hospital of Lanzhou University, Lanzhou, China.

Availability of data and materials: the datasets used and analyzed during the current study available from the corresponding author on reasonable request.

Funding: none.

Authors' contributions: the authors read and approved the manuscript for publication. QZ was a project administrator contributed to data curation, formal analysis, validation, and the literature review of the study. SM contributed to data curation, methodology, supervision, formal analysis, and the literature review of the study. XZ contributed to data curation, investigation, formal analysis, and the literature review of the study, review, and edited the manuscript for intellectual content. The authors agree to be accountable for all aspects of work ensuring integrity and accuracy.

Disclosure: no potential conflict of interest relevant to this article was reported. 


\section{REFERENCES}

1. Zhao Q, Ming J, Liu C, Shi L, Xu X, Nie X, et al. Multifocality and total tumor diameter predict central neck lymph node metastases in papillary thyroid microcarcinoma. Ann Surg Oncol. 2013;20:746-52.

2. Lai $X$, Zhang B, Jiang $Y$, Li J, Zhao R, Yang $X$, et al. Sonographic and Clinical Features of Papillary Thyroid Microcarcinoma Less than or Equal to Five Millimeters: A Retrospective Study. PLoS One. 2016;11:e0148567.

3. Ducoudray R, Tresallet C, Godiris-Petit G, Tissier F, Leenhardt L, Menegaux F. Prophylactic lymph node dissection in papillary thyroid carcinoma: Is there a place for lateral neck dissection? World J Surg. 2013;37:1584-91.

4. ItoY, Miyauchi A, Oda H. Low-risk papillary microcarcinoma of the thyroid: A review of active surveillance trials. Eur J Surg Oncol. 2018;44:307-15.

5. Lee HS, Park HS, Kim SW, Choi G, Park HS, Hong JC, et al. Clinical characteristics of papillary thyroid microcarcinoma less than or equal to $5 \mathrm{~mm}$ on ultrasonography. Eur Arch Otorhinolaryngol. 2013;270:2969-74.

6. Khokhar MT, Day KM, Sangal RB, Ahmedli NN, Pisharodi LR, Beland MD, et al. Preoperative high-resolution ultrasound for the assessment of malignant central compartment lymph nodes in papillary thyroid cancer. Thyroid. 2015;25:1351-4.

7. Takami H, Ito Y, Okamoto T, Yoshida A. Therapeutic strategy for differentiated thyroid carcinoma in Japan based on a newly established guideline managed by Japanese Society of Thyroid Surgeons and Japanese Association of Endocrine Surgeons. World J Surg. 2011;35:111-21.

8. Chinese Society of Clinical Oncology (CSCO) diagnosis and treatment guidelines for persistent/recurrent and metastatic differentiated thyroid cancer working group. Chinese Society of Clinical Oncology (CSCO) diagnosis and treatment guidelines for persistent/recurrent and metastatic differentiated thyroid cancer 2018 (English version). Chin J Cancer Res. 2019;31:99-116.

9. Feng JW, Yang $\mathrm{XH}, \mathrm{Wu}$ BO, Sun DL, Jiang $\mathrm{Y}, \mathrm{Qu} Z$ Z. Predictive factors for central lymph node and lateral cervical lymph node metastases in papillary thyroid carcinoma. Clin Transl Oncol. 2019;21:1482-91.

10. Oh HS, Kwon H, Song E, Jeon MJ, Song DE, Kim TY, et al. Preoperative clinical and sonographic predictors for lateral cervical lymph node metastases in sporadic medullary thyroid carcinoma. Thyroid. 2018;28:362-8.

11. Sitges-Serra A, Gallego-Otaegui L, Suarez S, Lorente-Poch L, Munne A, Sancho JJ. Inadvertent parathyroidectomy during total thyroidectomy and central neck dissection for papillary thyroid carcinoma. Surgery. 2017;161:712-9.

12. Vaisman F, Shaha A, Fish S, Michael Tuttle R. Initial therapy with either thyroid lobectomy or total thyroidectomy without radioactive iodine remnant ablation is associated with very low rates of structural disease recurrence in properly selected patients with differentiated thyroid cancer. Clin Endocrinol. 2011;75:112-9.

13. Furukawa MK, Furukawa M. Diagnosis of lymph node metastases of head and neck cancer and evaluation of effects of chemoradiotherapy using ultrasonography. Int J Clin Oncol. 2020;15:23-32.

14. American Thyroid Association (ATA) Guidelines Taskforce on Thyroid Nodules and Differentiated Thyroid Cancer, Cooper DS, Doherty GM, Haugen BR, Kloos RT, Lee SL, Mandel SJ, et al. Revised American Thyroid Association management guidelines for patients with thyroid nodules and differentiated thyroid cancer. Thyroid 2009;19:1167-214.

15. Vlassopoulou V, Vryonidou A, Paschou SA, loannidis D, Koletti A, Klonaris $\mathrm{N}$, et al. No considerable changes in papillary thyroid microcarcinoma characteristics over a 30-year time period. BMC Res Notes. 2016;9:1-9. 